\title{
Medium access prioritizing in the heterogeneous low-rate wireless PANs
}

\begin{abstract}
Coping with customer interests to employ Wireless Sensor Networks (WSN) in the new applications requires dealing with the fact of nodes variety that implies the heterogeneous nature of the traffic. This necessitates employing differentiation techniques that lead in the end to implementing prioritization in some processes of the system. The medium access controlling process plays a main role in the WSN work life cycle and the QoS provisioning is mainly based on the MAC efficiency. In this paper, we introduce the main techniques of WSN medium access prioritizing in the cluster-based Low-PANs. We propose using the prioritizing source as the categorizing factor. Furthermore, this study analyzes the impact of applying the MAC prioritization on the performance of the WSN. The simulation results prove that prioritizing nodes improves the system performance in terms of the access delay and the throughput metrics.
\end{abstract}

Keyword: Medium access control; Node-based; Priority; Traffic-based 